\title{
CALIBRATION AND TESTING OF A TLD DOSEMETER FOR AREA MONITORING
}

\author{
T. Buchillier ${ }^{1, *}$, A. Aroua ${ }^{1}$, F. Bochud ${ }^{1}$, C. Schuler ${ }^{2}$, N. Stritt ${ }^{3}$, J.-F. Valley ${ }^{1}$ and C. Wernli ${ }^{2}$ \\ ${ }^{1}$ Institut universitaire de radiophysique appliquée, Grand-Pré 1, CH-1007 Lausanne, Switzerland \\ ${ }^{2}$ Paul Scherrer Institute, CH-5232 Villigen PSI, Switzerland \\ ${ }^{3}$ Swiss Federal Office of Public Health, CH-3003 Bern, Switzerland
}

\begin{abstract}
The response of a TLD-600/TLD-700 area dosemeter has been characterized in neutron fields around the $590 \mathrm{MeV}$ cyclotron ring at the Paul Scherrer Institute (PSI). The dosemeter is based on a cylindrical paraffin moderator with three of each type of TLD chip at the centre, and is intended to use for area monitoring around accelerator facilities. The dosemeter is calibrated in terms of ambient dose equivalent using a non-moderated ${ }^{252} \mathrm{Cf}$ neutron source. The ambient dose equivalent response has been tested in five locations where the neutron fields and dose rates have been well characterized by Bonner sphere spectrometer and active neutron monitor measurements. The different spectrum shapes and dose rates in the five locations permit the comparison of the behavior of the active and passive dosemeters in these neutron fields.
\end{abstract}

\section{INTRODUCTION}

There is a practical application in area monitoring around accelerator facilities for a small, inexpensive, passive dosemeter, which is sensitive to neutrons, and that can be left unattended for several months and then easily exchanged and evaluated. According to ICRU 66, such a dosemeter should be calibrated in terms of ambient dose equivalent $H^{*}(10)^{(1)}$. Thermoluminescence dosemeters (TLDs) - widely used for personal dosimetry in photon and electron fields-have been envisaged for this purpose $e^{(1,2)}$.

Thermoluminescent materials with increased sensitivity for slow neutrons (e.g. through the $n\left({ }^{6} \mathrm{Li}, \alpha\right)$ ${ }^{3} \mathrm{H}$ reaction) are available. Alternatively, pairs of TLDs with one TLD sensitive to photons and neutrons, and the other TLD sensitive to photons only, can be used. Such detectors show a large variation in ambient dose equivalent response with neutron energy, and so a careful calibration based on the actual neutron spectrum to be measured is required for accurate measurements.

In this work, the usefulness of a passive TLD dosemeter for area monitoring is demonstrated, and a calibration method for the detector is described. The calibration uses ${ }^{252} \mathrm{Cf}$ beam measurements and on-site measurements around an accelerator in areas where the neutron fields have been well characterised by Bonner sphere spectrometer measurements.

Results from the passive TLD dosemeter are compared with measurements made using different active neutron dosemeters. The response of the active neutron dosemeters in similar neutron spectra was evaluated in an earlier study ${ }^{(3)}$.

*Corresponding author: Thierry.Buchillier@hospvd.ch

\section{MATERIALS AND METHODS}

\section{TLD dosemeter calibration}

The TLD dosemeter is contained within a polypropylene box of negligible thickness, and is comprised of a $110 \mathrm{~mm}$ diameter, by $120 \mathrm{~mm}$ long, cylindrical paraffin moderator, with a Perspex holder at the centre for three pairs of TLD-600/TLD-700 chips (Harshaw $3.2 \times 3.2 \times 0.9 \mathrm{~mm}^{3}{ }^{6} \mathrm{LiF}: \mathrm{Mg}, \mathrm{Ti}$ and $\left.{ }^{7} \mathrm{LiF}: \mathrm{Mg}, \mathrm{Ti}\right)$. The TLDs are easily exchanged onsite by removing the Perspex holder and replacing it with another holder with new chips in place. Chips are read out using a Harshaw 2000 TLD reader. Both types of TLD $\left({ }^{6} \mathrm{Li}\right.$ and $\left.{ }^{7} \mathrm{Li}\right)$ have a similar sensitivity to photons, but only ${ }^{6} \mathrm{Li}$ is sensitive to slow neutrons. Thus, the neutron dose equivalent is evaluated from the difference between the mean values of the readings for the three ${ }^{6} \mathrm{Li}$ and ${ }^{7} \mathrm{Li}$ chips.

The dosemeter calibration is performed in two steps. First, the individual sensitivities of the TLD chips are determined for ${ }^{60} \mathrm{Co}$ gamma radiation in terms of absorbed dose to water. The choice of this quantity is arbitrary, and permits the evaluation of the relative response of each chip, assuming that the relative sensitivities of the chips are the same for neutrons and photons. Then, the whole dosemeter (the paraffin moderator and the TLDs) is irradiated using the neutron field of the non-moderated ${ }^{252} \mathrm{Cf}$ source in the Paul Scherrer Institute (PSI) calibration laboratory. The reference dose equivalent is obtained from the laboratory's transfer standarda Berthold LB 6411 calibrated at the PhysikalischTechnische Bundesanstalt (PTB). Then the net signal from the TLDs (the difference between the absorbed dose measured with ${ }^{6} \mathrm{Li}$ and ${ }^{7} \mathrm{Li}$ chips) is compared with the reference dose equivalent to obtain a calibration factor expressed in $\mathrm{Sv} \mathrm{Gy}{ }^{-1}$. 


\section{T. BUCHILLIER ET AL}

The second part of the calibration is performed with two consecutive irradiations, both at a distance of $1 \mathrm{~m}$, in order to take into account scattered neutrons. First, at a reference ambient dose equivalent rate of $0.1 \mathrm{mSv} \mathrm{h}^{-1}$, without a shadow cone, and then with a shadow cone, at a reference ambient dose equivalent rate of $0.015 \mathrm{mSv} \mathrm{h}^{-1}$. The net TLD signal, corresponding to the primary neutron field alone, is used for the calibration.

\section{Bonner sphere spectrometer}

A Bonner sphere spectrometer was used to measure the neutron spectra and the reference dose levels in the neutron fields around the accelerator in which the TLD dosemeter was tested. This system consists of a ${ }^{3} \mathrm{He}$ proportional counter and a set of 11 polyethylene spheres with diameters ranging from 5 to $38 \mathrm{~cm}^{(4)}$. For high-energy neutrons, it was supplemented with ${ }^{209} \mathrm{Bi}$ and ${ }^{232}$ Th fission track detectors ${ }^{(5)}$.

\section{Active neutron monitors}

Ambient dose equivalent rates around the accelerator were also measured using several active neutron monitors; a Berthold LB 6411, a LINUS and an REM 500. The LB 6411 is a neutron detector with a $25 \mathrm{~cm}$ spherical moderator and a ${ }^{3} \mathrm{He} /$ methane proportional counter that is used in conjunction with the basic LB 1230 instrument. The energy dependence is $+10 \%$ to $-30 \%$ over the range $50 \mathrm{keV}$ to $10 \mathrm{MeV}^{(6)}$. The LINUS is based on a $\mathrm{BF}_{3}$ proportional counter and has a spherical moderator comprised of several layers of polyethylene, a boron-doped attenuator and a lead converter ${ }^{(7)}$. This configuration extends the response of this detector to several hundred MeV. The REM 500 (Health Physics Instruments) is a propane-filled, Rossi type, tissue-equivalent proportional counter (TEPC), with an energy range of $70 \mathrm{keV}$ to $20 \mathrm{MeV}^{(8)}$.

A total of five LB 6411 detectors (operated in integral mode) were used at each of the measurement locations to normalise the other instrument readings because the dose rate was varying with beam intensity and alignment. The measurements were performed over a period of about one month, during which the beam characteristics were kept constant, and the neutron spectra did not change appreciably.

\section{Measurement sites}

Measurements were made at five different sites in the experimental area of the accelerator. The sites were chosen to be representative of the areas in which workers would be occupationally exposed. The maximum dose equivalent rate for neutrons was $6 \mu \mathrm{Sv} \mathrm{h}^{-1}$. To limit the time required for the measurements, all the instruments were exposed simultanously. Site A was located in the vicinity of a carbon target behind several metres of concrete, site $\mathrm{B}$ in the accelerator area, site $\mathrm{C}$ in the building containing the $72 \mathrm{MeV}$ proton injector, site $\mathrm{D}$ was above the experimental hall (quite far from the experiment lines) and site E was near a workshop, also relatively far away from the accelerator beam and targets.

\section{RESULTS}

\section{Neutron spectra}

Bonner sphere and fission track detector readings, normalised to the monitoring instrument, were unfolded with the system response matrix to obtain the neutron spectra. An adapted version of the SAND unfolding code ${ }^{(9)}$ was used, starting with a uniform initial guess spectrum. The spectra obtained when starting with a fission initial spectrum were very similar. Figure 1 shows the neutron fluence spectra at sites $\mathrm{A}-\mathrm{E}$ in the lethargy representation.

The ambient dose equivalent rate $\dot{H}^{*}(10)$ and the personal dose equivalent rate for normal incidence on a slab phantom $\dot{H}_{\mathrm{p}}\left(10,0^{\circ}\right)$ were calculated from the neutron fluence spectra using fluence-to-dose conversion coefficients from ICRP $74^{(10)}$. Table 1 shows the dosimetric quantities, with expanded uncertainties estimated at $20 \% \quad(95 \%$ confidence level) on the basis of calibrations and intercomparisons, in addition to the statistical counting uncertainties.

\section{TLD dosemeter calibration factor}

The TLD dosemeter calibration factor obtained from the ${ }^{252} \mathrm{Cf}$ beam calibration is $0.477 \mathrm{~Sv} \mathrm{~Gy}{ }^{-1}$. The expanded uncertainty ( $95 \%$ confidence level) is evaluated at $7 \%$, with the main components; reproducibility $(5 \%)$, and the transfer of the reference standard $(5 \%)$.

The ambient dose equivalent response of the TLD dosemeter for a given measurement site is obtained from the ratio of $H^{*}(10)$ measured using the dosemeter, to $H^{*}(10)$ obtained from the Bonner sphere spectrometer applying fluence-to-dose conversion coefficients (Table 2). Table 2 also shows the count rate ratio of the 9 and 3 inch spheres as an index of neutron field hardness ${ }^{(11)}$. Figure 2 shows the response of the TLD dosemeter as a function of this index.

The detection limit of the TLD dosemeter was assessed using the results of quarterly evaluations of the dosemeter, exposed to environmental background radiation, over the course of $1 \mathrm{y}$. The 

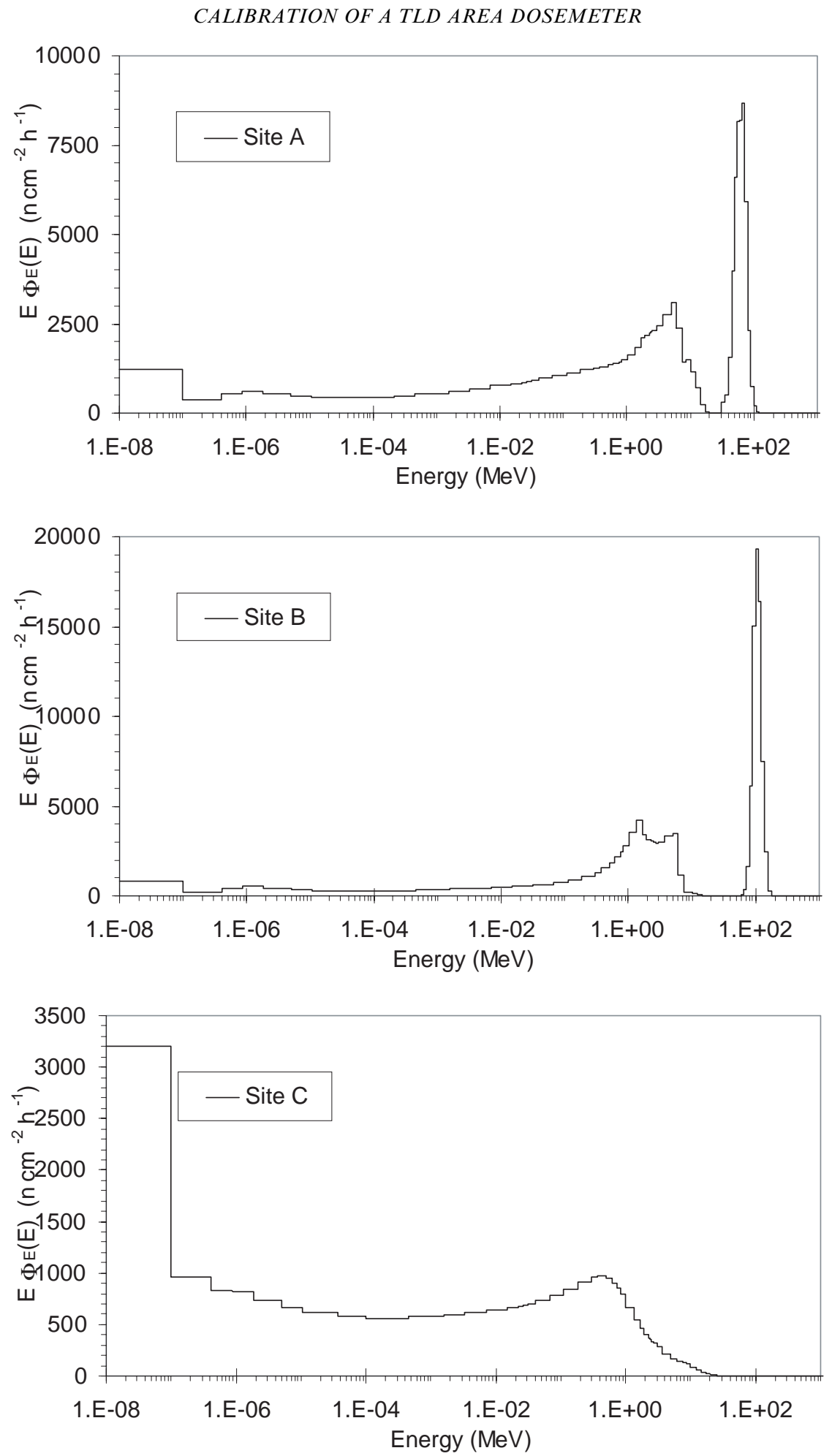

Figure 1. Fluence spectra at the five sites. 

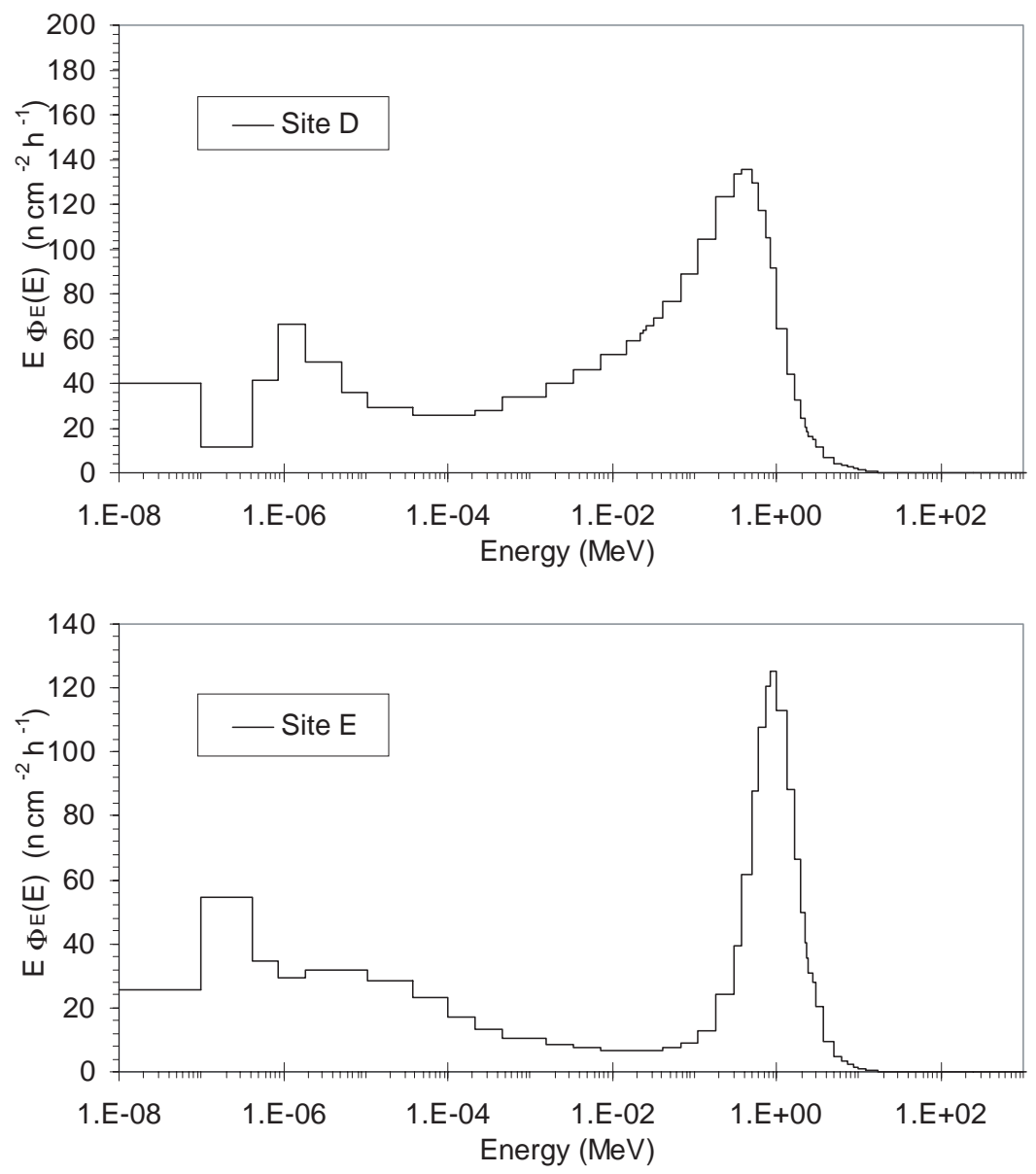

Figure 1. Continued.

Table 1. Dosimetric quantities calculated from the neutron fluence spectra.

\begin{tabular}{lccccc}
\hline Quantity & \multicolumn{5}{c}{ Site } \\
\cline { 2 - 6 } & $\mathrm{A}$ & $\mathrm{B}$ & $\mathrm{C}$ & $\mathrm{D}$ & $\mathrm{E}$ \\
\hline$\dot{\phi}\left(\mathrm{n} \mathrm{cm}^{-2} \mathrm{~s}^{-1}\right)$ & 6.78 & 6.44 & 6.17 & 0.28 & 0.16 \\
$\dot{H}^{*}(10)\left(\mu \mathrm{Sv} \mathrm{h} \mathrm{h}^{-1}\right)$ & 4.81 & 5.42 & 1.11 & 0.098 & 0.076 \\
$\dot{H}_{p}\left(10,0^{\circ}\right)\left(\mu \mathrm{Sv} \mathrm{h}^{-1}\right)$ & 4.94 & 5.57 & 1.17 & 0.102 & 0.078 \\
Expanded uncert. (\%) & 30 & 30 & 22 & 26 & 36 \\
\hline
\end{tabular}

measured neutron dose equivalents (mean value $0.02 \mathrm{mSv})$ and the standard deviation $(0.01 \mathrm{mSv})$ of the quarterly readings were compared with the published neutron effective dose in areas with normal background $(0.014 \mathrm{mSv})^{(12)}$. Using this method, the detection limit of the dosemeter is estimated to be $0.03 \mathrm{mSv}$ for a three month measurement period.

\section{Active neutron monitors}

The three active neutron monitors provided direct values for the dose equivalent rate. Table 2 shows the relative responses of the active monitors, normalised to the Bonner sphere spectrometer measurements. The combined standard uncertainties can be attributed to the calibration uncertainties for the monitors ( $5 \%$ at $95 \%$ confidence level), either as stated in the manufacturer's specification, or as estimated after verification in the calibration laboratory, and to the significant statistical counting uncertainties present 
CALIBRATION OF A TLD AREA DOSEMETER

Table 2. Ambient dose equivalent response of TLD dosemeter and active dosemeters normalised to the Bonner sphere spectrometer (BS).

\begin{tabular}{lcccccc}
\hline Site & $9 / 3$ inch ratio & BS & TLD & LB 6411 & LINUS & REM 500 \\
\hline A & 0.79 & 1.0 & $1.41 \pm 0.23$ & $0.86 \pm 0.13$ & $0.74 \pm 0.12$ & $1.29 \pm 0.21$ \\
B & 1.17 & 1.0 & $0.86 \pm 0.14$ & $0.70 \pm 0.10$ & $1.21 \pm 0.19$ & $1.36 \pm 0.22$ \\
C & 0.27 & 1.0 & $2.21 \pm 0.29$ & $1.05 \pm 0.12$ & $1.11 \pm 0.13$ & $0.75 \pm 0.10$ \\
D & 0.20 & 1.0 & $2.07 \pm 0.29$ & $1.00 \pm 0.13$ & $0.97 \pm 0.14$ & $1.62 \pm 0.27$ \\
E & 0.49 & 1.0 & $1.45 \pm 0.27$ & $0.54 \pm 0.10$ & n/a & $0.53 \pm 0.12$ \\
\hline
\end{tabular}

Uncertainties are given at the $95 \%$ confidence level. n/a not available.

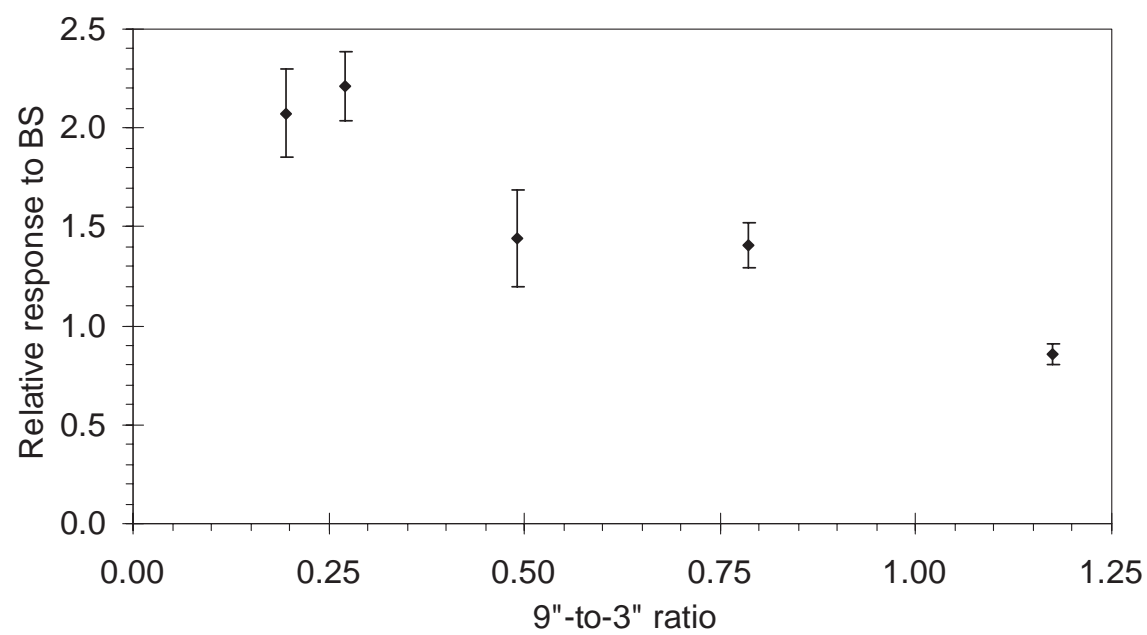

Figure 2. TLD dosemeter relative response to BS in function of the 9- to 3-inch ratio.

at low dose rates. The uncertainties do not include the contribution of the systematic uncertainty of the reference instrument.

\section{DISCUSSION}

\section{Neutron spectra}

The lethargy spectrum A is characterised by a highenergy peak at $50 \mathrm{MeV}$, and a second peak $\sim 5 \mathrm{MeV}$ (evaporation peak). The same characteristics are present in the lethargy spectrum B at 100 and $2 \mathrm{MeV}$.

At sites $\mathrm{C}, \mathrm{D}$ and $\mathrm{E}$, no significant amount of tracks were observed on the $\mathrm{Th} / \mathrm{Bi}$ detectors, and the unfolding is based on the Bonner sphere measurements only. At site $\mathrm{C}$ (close to the injector), the lethargy spectrum contains dominant thermal and intermediate contributions. The lethargy spectrum D shows mainly intermediate neutrons with a much lower fluence, as does the lethargy spectrum E, with an even lower neutron fluence.

\section{Dosimetric results}

The ambient dose equivalents measured using the TLD dosemeter calibrated in this work agree well with the values obtained from spectrometry applying fluence-to-dose coefficients (reference value) for three of the sites, and do not agree at the other two sites. The relative response of the detector is close to unity for high energy, fast neutron spectra, and reaches a factor of 2.2 at thermal and intermediate neutron energies. The response of the detector at site $\mathrm{E}$ is difficult to ascertain because of large statistical uncertainties.

The relative response of the detector established in this work can be used to correct the readings according to the type of neutron spectrum. Thus, provided that approximate information is available about the neutron energy, this simple passive dosemeter can be used for routine monitoring around proton accelerators.

The detection limit of $30 \mu \mathrm{Sv}$ over a three month period is adequate, since the observed dose rates 


\section{T. BUCHILLIER ET AL.}

of $0.08-6 \mu \mathrm{Sv} \mathrm{h}^{-1}$, led to doses in the range $160 \mu \mathrm{Sv}$ to $12 \mathrm{mSv}$ over three months. Under normal environmental background conditions, the dose rate (typically $6 \mathrm{nSv} \mathrm{h}^{-1}$ ) would not be measurable. A measuring instrument with sensitivity at least three times higher would be necessary for environmental background measurements.

The active dosemeters show better agreement than the TLD dosemeter with the spectrometer results. Most of the instruments agree within the measurement uncertainty, but some discrepancies are observed. The LB 6411 slightly underestimates dose at high energies (above its specified range), and also at site $\mathrm{E}$ (for an unknown reason). The discrepancy of the LINUS at site A is difficult to explain because the features of this instrument make it suitable for a large energy range. At the other sites, there is good agreement. The REM 500 overestimates dose for spectrum $C$ with thermal and intermediate neutrons. The underestimation of the dose at site E may originate from the larger uncertainty of this TEPC due to the poor statistics. It is difficult to estimate the uncertainty in the LET spectrum used for dose calculation. The response of the active dosemeters, in neutron spectra similar to those used in this study, was characterised in an earlier study ${ }^{(3)}$. The uncertainties for this study are higher (lower dose rates, instruments not exposed at exactly the same locations), but the results are consistent with the earlier findings.

\section{CONCLUSIONS}

A passive TLD dosemeter has been calibrated for routine monitoring of ambient dose equivalent, and tested in realistic conditions by comparing with neutron spectra results. Active neutron monitor dose rate measurements differ from the TLD dosemeter measurements by up to a factor of 2. The TLD dosemeter is more accurate when the type of neutron spectrum is known, and its response is corrected by taking into account the relative response determined in this work. This is consistent with the findings of Dinter et $a l .{ }^{(2)}$ for a similar TLD.

\section{ACKNOWLEDGEMENTS}

The authors wish to thank R. Porta, M. Boschung, O. Morath and P. Wyrsch for their assistance during the measurements, and gratefully acknowledge the support of the Swiss Federal Office of Public Health for this work.

\section{REFERENCES}

1. International Commission on Radiation Units and Measurements. Determination of operational dose equivalent quantities for neutrons. ICRU Report 66. J. ICRU 1(3) (Ashford: Nuclear Technology Publishing) (2001), ISBN 1870965922.

2. Dinter, H., Racky, B. and Tesch, K. Neutron dosimetry at high energy accelerators. Nucl. Instrum. Meth. A 376, 104-114 (1996).

3. Grecescu, M., Aroua, A., Boschung, M., Fiechtner, A., Gmür, K., Laedermann, J.-P., Valley, J.-F. and Wernli, C. Neutron field measurements at the $590 \mathrm{MeV}$ ring cyclotron of the Paul Scherrer Institute. Nucl. Instrum. Meth. A 476, 58-62 (2002).

4. Aroua, A., Gecescu, M., Lerch, P., Prêtre, S., Valley, J.-F. and Vylet, V. Evaluation and test of the response matrix of a multisphere neutron spectrometer in a wide energy range. Nucl. Instrum. Meth. A 321, 298-316 (1992).

5. Aroua, A. High energy extension of a neutron spectrometer by means of thorium and bismuth fission detectors. Technical Memorandum CERN/TIS-RP/ TM/93-18, CERN, Geneva (1993).

6. Burgkhardt, B., Fieg, G., Klett, A., Plewnia, A. and Siebert, B. R. L. The neutron fluence and $H^{*}(10)$ response of the new LB 6411 rem counter. Radiat. Prot. Dosim. 70(1-4), 361-364 (1997).

7. Birattari, C., Esposito, A., Ferrari, A., Pelliccioni, M., Rancati, T. and Silari, M. The extended range rem counter "LINUS": overview and latest developments. Radiat. Prot. Dosim. 76(3), 135-138 (1998).

8. Health Physics Instruments. Model REM-500 Neutron Survey Meter. Operation and repair manual (1993).

9. Vylet, V. Détermination des champs de radiations neutroniques par la méthode des sphères de Bonner. Ph.D. thesis No. 671, Ecole Polytechnique Fédérale de Lausanne, Lausanne (1987).

10. International Commission on Radiological Protection. Conversion coefficients for use in radiological protection against external radiation. ICRP Publication 74 . Ann. ICRP 26(3) (Oxford: Pergamon Press) (1996).

11. Aroua, A., Grecescu, M., Prêtre, S. and Valley, J.-F. On the use of some spectrum hardness quantifiers for operational neutron fields. Radiat. Prot. Dosim. 54(2), 99-104 (1994).

12. Thompson, I. M. G. Technical recommendations on measurements of external environmental gamma radiation doses. A report of EURADOS working group 12 'Environmental radiation monitoring'. Radiat. Prot. Dosim. 92(1-3), 71-76 (2000). 